Also available at http://amc-journal.eu

ISSN 1855-3966 (printed edn.), ISSN 1855-3974 (electronic edn.)

ARS MATHEMATICA CONTEMPORANEA 14 (2018) 329-344

\title{
A note on the thickness of some complete bipartite graphs
}

\author{
Siwei $\mathrm{Hu}$, Yichao Chen * \\ Department of Mathematics, Hunan University, 410082 Changsha, China
}

Received 22 November 2016, accepted 17 July 2017, published online 19 September 2017

\begin{abstract}
The thickness of a graph is the minimum number of planar subgraphs into which the graph can be decomposed. Determining the thickness for the complete bipartite graph is an unsolved problem in graph theory for over fifty years. Using a new planar decomposition for $K_{4 k-4,4 k}(k \geq 4)$, we obtain the thickness of the complete bipartite graph $K_{n, n+4}$, for $n \geq 1$.
\end{abstract}

Keywords: Planar graph, thickness, complete bipartite graph.

Math. Subj. Class.: 05C10

\section{Introduction}

In this paper, all graphs are simple. A graph $G$ is denoted by $G=(V, E)$ where $V(G)$ is the vertex set and $E(G)$ is the edge set. A complete graph is a graph in which any two vertices are adjacent. A complete graph on $n$ vertices is denoted by $K_{n}$. A complete bipartite graph is a graph whose vertex set can be partitioned into 2 parts, such that every edge has its ends in different parts and every two vertices in different parts are adjacent. We use $K_{p_{1}, p_{2}}$ to denote a complete bipartite graph in which the $i^{\text {th }}$ part contains $p_{i}$ vertices, for $i=1,2$.

The thickness $t(G)$ of a graph $G$ is the minimum number of planar subgraphs into which $G$ can be decomposed [15]. It is a classical topological parameter of a graph and has many applications, for instance, to graph drawing [3] and VLSI design [1]. Since deciding the thickness of a graph is NP-hard [11], it is very difficult to get the exact number of thickness for arbitrary graphs. Battle, Harary and Kodama [4] in 1962 and Tutte [14] in 1963 independently showed that the thickness of $K_{9}$ and $K_{10}$ equals 3 . Beineke and Harary [5] determined the thickness of complete graph $K_{n}$ for $n \not \equiv 4(\bmod 6)$ in 1965 , the remaining case was solved in 1976, independently by V.B. Alekseev and V.S. Gonchakov [2] and by J.M. Vasak [13].

\footnotetext{
* Corresponding author.

E-mail addresses: husiwei@hnu.edu.cn (Siwei Hu), ycchen@hnu.edu.cn (Yichao Chen)
} 
For complete bipartite graphs, the problem has not been entirely solved yet. By constructing a planar decomposition of $K_{m, n}$ when $m$ is even, Beineke, Harary and Moon [6] determined the thickness of $K_{m, n}$ for most values of $m, n$ in 1964.

Theorem 1.1. [6] For $m \leq n$, the thickness of the complete bipartite graph $K_{m, n}$ is

$$
t\left(K_{m, n}\right)=\left\lceil\frac{m n}{2(m+n-2)}\right\rceil,
$$

except possibly when $m$ and $n$ are both odd and there exists an integer $k$ satisfying $n=$ $\left\lfloor\frac{2 k(m-2)}{(m-2 k)}\right\rfloor$.

We recall that the thickness of $K_{n, n}$ is also obtained in 1968 by Isao and Ozaki [10] independently. The following open problem is adapted from [8] by Gross and Harary.

Problem 1.2. [See Problem 4.1 of [8]] Find the thickness of $K_{m, n}$ for all $m, n$.

Beineke, Harary and Moon [6] also pointed out that the smallest complete bipartite graph whose thickness is unknown is $K_{17,21}$. From Euler's Formula, the thickness of $K_{17,21}$ is at least 5 .

From Theorem 1.1, we need to determine the thickness of $K_{m, n}$ for odd $m, n$. Since the difference between the two odd numbers is even, we only need to determine the thickness of $K_{n, n+2 k}$ for odd $n$ and $k \geq 0$. In this paper, we start to calculate the thickness of $K_{n, n+2 k}$ for some small values of $k$. Indeed, we determine the thickness of $K_{n, n+4}$.

Theorem 1.3. The thickness of $K_{n, n+4}$ is

$$
t\left(K_{n, n+4}\right)= \begin{cases}1, & \text { if } n \leq 2 \\ \left\lceil\frac{n+3}{4}\right\rceil, & \text { otherwise. }\end{cases}
$$

The following corollary follows from Theorem 1.3.

Corollary 1.4. The thickness of $K_{17,21}$ is 5 .

We may refer the reader to $[7,12,16]$ for more background on graph thickness.

\section{The thickness of $K_{n, n+4}$}

To begin with, we define two special graphs called the pattern graph and the $k^{\text {th }}$-order nest graph. Then, we prove a new planar decomposition of $K_{4 k-4,4 k}$. Finally, we prove the thickness of $K_{4 k-3,4 k+1}$ and $K_{n, n+4}$.

\subsection{The pattern graph}

Let $U=\left\{u_{1}, u_{2}\right\}$ and $X_{n}$ be a set of $n$ vertices. A graph is said to be a pattern graph of order $n+2$, denoted by $G\left[u_{1} X_{n} u_{2}\right]$, if it can be constructed by the following two steps.

1. Arrange the $n$ vertices in a row, and put vertices $u_{1}, u_{2}$ on the above and below of $n$ vertices, respectively.

2. Join both $u_{1}$ and $u_{2}$ to the $n$ vertices using straight lines. 
From the definition above, the pattern graph is a planar straight-line graph. Figure 1 illustrates the pattern graph $G\left[u_{1} X_{n} u_{2}\right]$.

Remark 2.1. Unless explicitly mentioned, we always join vertices using straight lines in the drawings of the following proofs.

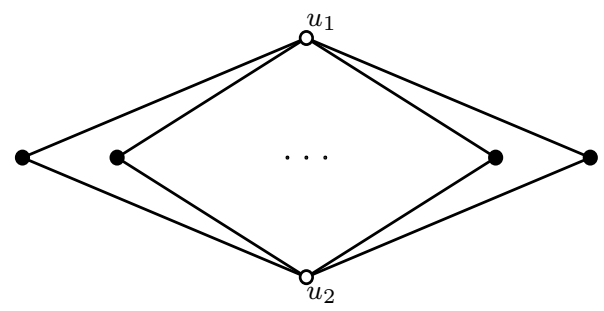

Figure 1: The pattern graph $G\left[u_{1} X_{n} u_{2}\right]$.

\subsection{The $k^{t h}$-order nest graph}

Let $U_{k}=\left\{u_{i_{1}}, u_{i_{2}}, \ldots, u_{i_{k}}\right\}, V_{k}=\left\{v_{j_{1}}, v_{j_{2}}, \ldots, v_{j_{k}}\right\}$ and $W_{2 k+2}=\left\{w_{l_{1}}, w_{l_{2}}, \ldots\right.$, $\left.w_{l_{2 k+2}}\right\}$, we define a $k^{t h}$-order nest graph $G\left[U_{k}, V_{k}, W_{2 k+2}\right]$ as follows:

1. Arrange $2 k+2$ vertices $w_{l_{1}}, w_{l_{2}}, \ldots, w_{l_{2 k+2}}$ in a row.

2. For $1 \leq m \leq k$, place vertices $u_{i_{m}}$ and $v_{j_{m}}$ on the above and below of the row, respectively, and join them to $w_{l_{1}}, w_{l_{2 m}}, w_{l_{2 m+1}}, w_{l_{2 m+2}}$.

Figure 2 illustrates a third-order nest graph $G\left[U_{3}, V_{3}, W_{8}\right]$, where $U_{3}=\left\{u_{1}, u_{2}, u_{3}\right\}$, $V_{3}=\left\{v_{1}, v_{2}, v_{3}\right\}$ and $W_{8}=\left\{w_{1}, w_{2}, \ldots, w_{8}\right\}$.

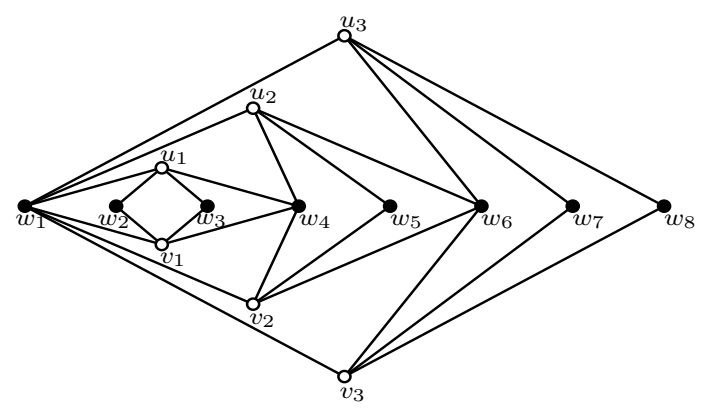

Figure 2: The third-order nest graph $G\left[U_{3}, V_{3}, W_{8}\right]$.

\subsection{A new planar decomposition of $K_{4 k-3,4 k+1}$, for $k \geq 4$}

In this subsection, we shall construct a planar decomposition for the complete bipartite graph $K_{4 k-3,4 k+1}$ with $k$ planar subgraphs $G_{1}, G_{2}, \ldots, G_{k}$. Suppose that the vertex partition of $K_{4 k-3,4 k+1}$ is $(X, Y)$, where $X=\left\{x_{1}, x_{2}, \ldots, x_{4 k-3}\right\}, Y=\left\{y_{0}, y_{1}, y_{2}, \ldots, y_{4 k}\right\}$. 


\subsubsection{The planar decomposition for $K_{4 k-4,4 k}$}

Let the vertex partition of $K_{4 k-4,4 k}$ be $\left(X_{1}, Y_{1}\right)$, where $X_{1}=\left\{x_{1}, x_{2}, \ldots, x_{4 k-4}\right\}, Y_{1}=$ $\left\{y_{0}, y_{1}, \ldots, y_{4 k-1}\right\}$. In this subsection, all subscripts in $y_{j}$ are taken $\bmod 4 k$.

1. In the graph $G_{i}(1 \leq i \leq k)$, we arrange $4 k$ vertices in a row, and divide the $4 k$ vertices into two subsets $L_{2 k}$ and $R_{2 k}$ such that each subset contains $2 k$ vertices according to the following steps.

2. In the graph $G_{i}(1 \leq i \leq k-1)$, we choose four vertices $x_{4 i-3}, x_{4 i-2}, x_{4 i-1}, x_{4 i}$ from $X_{1}$ and construct two pattern graphs $G\left[x_{4 i-3} L_{2 k} x_{4 i-1}\right]$ and $G\left[x_{4 i-2} R_{2 k} x_{4 i}\right]$. Then we join both $x_{4 i-3}$ and $x_{4 i-1}$ to the first vertex and the last vertex in $R_{2 k}$. Finally, we label the vertices in $L_{2 k}$ and $R_{2 k}$ as $y_{1}, y_{3}, y_{5}, \ldots, y_{4 k-1}$ and $y_{2 i+6}, y_{2 i+8}$, $y_{2 i+10}, \ldots, y_{2 i+4 k+4}$ in turn, respectively.

3. In the graph $G_{k}$, we label the vertices in $L_{2 k}$ and $R_{2 k}$ as $y_{1}, y_{3}, y_{5}, \ldots y_{4 k-1}$ and $y_{2}, y_{4}, \ldots, y_{4 k-2}, y_{0}$, respectively. First, we construct a $(k-1)^{t h}$-order nest graph $G\left[U_{k-1}, V_{k-1}, W_{2 k}\right]$, where $U_{k-1}=\left\{x_{2}, x_{6}, x_{10}, \ldots, x_{4 k-6}\right\}, V_{k-1}=\left\{x_{4}, x_{8}\right.$, $\left.x_{12}, \ldots, x_{4 k-4},\right\}$ and $W_{2 k}=\left\{y_{1}, y_{3}, y_{5}, \ldots, y_{4 k-1}\right\}$. We join $x_{4 i-3}$ to $y_{2 i}$ and $y_{2 i+2}$, for $1 \leq i \leq k-1$. Second, we construct a union of paths, if $k$ is even, we join $x_{4 i-1}$ to $y_{2 i+2 k}$ and $y_{2 i+2+2 k}$, for $1 \leq i \leq k-1$; otherwise $k$ is odd, we join $x_{4 i-1}$ to $y_{2 i+2 k-2}$ and $y_{2 i+2 k}$, for $1 \leq i \leq k-1$.

4. In each graph $G_{j}(1 \leq j \leq k-1)$, we put $x_{4 i-2}, x_{4 i}$ in the quadrangle $x_{4 j-3} y_{4 j+1}$ $x_{4 j-1} y_{4 j+3}$, and join them to $y_{4 j+1}$ and $y_{4 j+3}$, for $1 \leq i<j$. We put the vertices $x_{4 i-2}, x_{4 i}$ in the quadrangle $x_{4 j-3} y_{4 j-1} x_{4 j-1} y_{4 j+1}$, and join both $x_{4 i-2}$ and $x_{4 i}$ to $y_{4 j-1}$ and $y_{4 j+1}$, for $j<i \leq k-1$. Next, we put $x_{4 i-3}$ in the quadrangle $x_{4 j-2} y_{4 j-2 i+4} x_{4 j} y_{4 j-2 i+6}$, and join $x_{4 i-3}$ to $y_{4 j-2 i+4}, y_{4 j-2 i+6}$, for $1 \leq i<j$. We put $x_{4 i-3}$ in the quadrangle $x_{4 j-2} y_{4 j-2 i+4 k} x_{4 j} y_{4 j-2 i+4 k+2}$, and join $x_{4 i-3}$ to $y_{4 j-2 i+4 k}, y_{4 j-2 i+4 k+2}$, for $j<i \leq k-1$.

For each $i(1 \leq i \leq k-1)$, we define a set $M_{i}=\{i+1, i+2, \ldots, i+k-2\}$. Suppose that $m \in M_{i}$, if $m \leq k-1$, we let $j=m$; otherwise, $j=m-k+1$.

(i) $k$ is even. If $i+1 \leq m \leq i+\frac{k-4}{2}$, we put $x_{4 i-1}$ in the quadrangle $x_{4 j-2} y_{4 m-2 i+4}$ $x_{4 j} y_{4 m-2 i+6}$, and join $x_{4 i-1}$ to $y_{4 m-2 i+4}, y_{4 m-2 i+6}$. If $i+\frac{k-4}{2}+1 \leq m \leq i+k-2$, we put $x_{4 i-1}$ in the quadrangle $x_{4 j-2} y_{4 m-2 i+8} x_{4 j} y_{4 m-2 i+10}$, and join $x_{4 i-1}$ to $y_{4 m-2 i+8}, y_{4 m-2 i+10}$.

(ii) $k$ is odd. If $i+1 \leq m \leq i+\frac{k-5}{2}$, we put $x_{4 i-1}$ in the quadrangle $x_{4 j-2} y_{4 m-2 i+4}$ $x_{4 j} y_{4 m-2 i+6}$, and join $x_{4 i-1}$ to $y_{4 m-2 i+4}, y_{4 m-2 i+6}$. If $i+\frac{k-5}{2}+1 \leq m \leq i+k-2$, we put $x_{4 i-1}$ in the quadrangle $x_{4 j-2} y_{4 m-2 i+8} x_{4 j} y_{4 m-2 i+10}$, and join $x_{4 i-1}$ to $y_{4 m-2 i+8}, y_{4 m-2 i+10}$.

Theorem 2.2. Let $G_{1}, G_{2}, \ldots, G_{k}$ be the planar subgraphs obtained from steps 1, 2, 3 and 4 above, then $\left\{G_{1}, G_{2}, \ldots, G_{k}\right\}$ is a planar decomposition of $K_{4 k-4,4 k}$.

Proof. From the constructions above, we have $E\left(G_{i}\right) \cap E\left(G_{j}\right)=\emptyset$, for $1 \leq i \neq j \leq k$. In order to prove that $\left\{G_{1}, G_{2}, \ldots, G_{k}\right\}$ is a planar decomposition of $K_{4 k-4,4 k}$, we need to show that $E\left(G_{1}\right) \cup E\left(G_{2}\right) \cup \cdots \cup E\left(G_{k}\right)=E\left(K_{4 k-4,4 k}\right)$. We denote $d_{G_{i}}(v)$ as the degree of $v$ in $G_{i}$, for $1 \leq i \leq k$. 
By the construction above, Step 2 contributes to the degrees of $v_{4 i-3}, v_{4 i-1}, v_{4 i-2}$, and $v_{4 i}$ in $G_{i}$ by terms $2 k+2,2 k+2,2 k+1$ and $2 k+1$, respectively. In other words, we have $d_{G_{i}}\left(v_{4 i-3}\right)=d_{G_{i}}\left(v_{4 i-1}\right)=2 k+2$ and $d_{G_{i}}\left(v_{4 i-2}\right)=d_{G_{i}}\left(v_{4 i}\right)=2 k+1$.

For $1 \leq i \leq k-1$, Step 3 contributes to $d_{G_{k}}\left(v_{4 i-3}\right), d_{G_{k}}\left(v_{4 i-1}\right), d_{G_{k}}\left(v_{4 i-2}\right)$ and $d_{G_{k}}\left(v_{4 i}\right)$ by terms $2,2,3$, and 3 , respectively.

For $1 \leq j \leq k-1$ and $i \neq j$, Step 4 contributes to each of $d_{G_{j}}\left(v_{4 i-3}\right), d_{G_{j}}\left(v_{4 i-1}\right)$, $d_{G_{j}}\left(v_{4 i-2}\right)$ and $d_{G_{j}}\left(v_{4 i}\right)$ a term 2 .

In total, for $1 \leq i \leq k-1$, we have

$\sum_{j=1}^{k} d_{G_{j}}\left(v_{4 i-1}\right)=\sum_{j=1}^{k} d_{G_{j}}\left(v_{4 i-3}\right)=d_{G_{i}}\left(v_{4 i-3}\right)+\sum_{1 \leq j \neq i \leq k-1}^{k} d_{G_{j}}\left(v_{4 i-3}\right)+d_{G_{k}}\left(v_{4 i-3}\right)$ $=2 k+2+2(k-2)+2=4 k$,

and $\sum_{j=1}^{k} d_{G_{j}}\left(v_{4 i-2}\right)=\sum_{j=1}^{k} d_{G_{j}}\left(v_{4 i}\right)=d_{G_{i}}\left(v_{4 i}\right)+\sum_{1 \leq j \neq i \leq k-1}^{k} d_{G_{j}}\left(v_{4 i}\right)+d_{G_{k}}\left(v_{4 i}\right)=$ $2 k+1+2(k-2)+3=4 k$.

From the discussion above, the result follows.

\subsubsection{Add the vertex $x_{4 k-3}$}

1. In the graph $G_{i}(1 \leq i \leq k-1)$, put the vertex $x_{4 k-3}$ in the quadrangle $x_{4 i-3} y_{4 i-1}$ $x_{4 i-1} y_{4 i+1}$, and join it to $y_{4 i-1}, y_{4 i+1}$.

2. In the graph $G_{k}$, place the vertex $x_{4 k-3}$ below the row of $2 k$ vertices of $R_{2 k}$, and join it to $y_{1}, y_{4 k-1}$ and all the $2 k$ vertices of $R_{2 k}$.

\subsubsection{Add the vertex $y_{4 k}$}

1. In the graph $G_{i}(1 \leq i \leq k-1)$, put the vertex $y_{4 k}$ in the quadrangle $x_{4 i-2} y_{4 i+8} x_{4 i}$ $y_{4 i+10}$, and connect it to $x_{4 i-2}, x_{4 i}$.

2. In the graph $G_{k}$, place the vertex $y_{4 k}$ above the row of vertices of $R_{2 k}$, and join it to $x_{1}, x_{5}, \ldots, x_{4 k-7}, x_{3}, x_{7}, \ldots, x_{4 k-3}$.

We have the following theorem.

Theorem 2.3. The thickness of $K_{4 k-3,4 k+1}$ is $k$, for $k \geq 4$.

Proof. From Theorem 2.2, Subsection 2.3.2 and Subsection 2.3.3, a planar decomposition of $K_{4 k-3,4 k+1}$ with $k$ planar subgraphs $G_{1}, G_{2}, \ldots, G_{k}$ is obtained. From Euler's formula, we have

$$
t\left(K_{4 k-3,4 k+1}\right) \geq\left\lceil\frac{(4 k-3)(4 k+1)}{2(8 k-4)}\right\rceil=k,
$$

and so $t\left(K_{4 k-3,4 k+1}\right)=k$.

Example 2.4. By using the procedure above, the two planar decompositions of $K_{17,21}$ ( $k=5$ is odd) and $K_{21,25}(k=6$ is even) are shown in Appendix A (See Figures 3-7) and Appendix B (See Figures 8-13), respectively. 


\subsection{Proof of Theorem 1.3}

From Theorem 1.1, the proof has two cases:

Case 1: $n=4 k-3(k>0)$. When $1 \leq k \leq 3$, it is routine to check that the theorem is true. For $k \geq 4,\left\lfloor\frac{2 k(4 k-3-2)}{4 k-3-2 k}\right\rfloor=\left\lfloor 4 k+1+\frac{3}{2 k-3}\right\rfloor=4 k+1$, thus, the thickness of $K_{4 k-3,4 k+1}$ can not be determined by Theorem 1.1. By Theorem 2.3, we have $t\left(K_{4 k-3,4 k+1}\right)=k=$ $\left\lceil\frac{n+3}{4}\right\rceil$.

Case 2: $n=4 k-1(k>0)$. Since $4 k-1$ and $4 k+3$ are both odd and $4 k+3 \neq$ $\left\lfloor\frac{2(k+1)(4 k-1-2)}{4 k-1-2(k+1)}\right\rfloor$ (See Lemma 1 of [6] for details), the thickness of $K_{4 k-1,4 k+3}$ can be determined by Theorem 1.1, thus

$$
\begin{aligned}
t\left(K_{n, n+4}\right)=t\left(k_{4 k-1,4 k+3}\right) & =\left\lceil\frac{(4 k-1)(4 k+3)}{2(4 k-1+4 k+3-2)}\right\rceil \\
& =\left\lceil k+\frac{1}{2}-\frac{3}{16 k}\right\rceil=k+1=\left\lceil\frac{n+3}{4}\right\rceil
\end{aligned}
$$

Summarizing the above, the theorem follows.

\section{Conclusion}

In this paper, we determine the thickness for $K_{n, n+4}$. The proof replies on a planar decomposition of $K_{4 k-3,4 k+1}$ and the Theorem 1.1 of Beineke, Harary and Moon. We observe that our approach for the construction of a planar decomposition of $K_{n, n+4}$ is the first step in finding a solution for Problem 1.2. From Theorem 1.1, the next classes of complete bipartite graphs whose thickness is unknown is $K_{4 k-1,4 k+7}$, for $k \geq 10$. Furthermore, the new smallest complete bipartite graph whose thickness is unknown is $K_{19,29}$. We hope that the construction here helps establish intuition and structure of the Problem 1.2.

Another way of solving the Problem 1.2 is to find a new planar decomposition of $K_{m, n}$, for odd $m, n$. Actually, using a new planar decomposition of the complete tripartite graph $K_{1, g, n}$ and a recursive construction, we also [9] obtained the thickness of $K_{s, t}$, where $s$ is odd and $t \geq \frac{(s-3)(s-2)}{3}$. Now we split Problem 1.2 into the following two problems.

Problem 3.1. Find the thickness of $K_{n, n+4 k}$ for odd $n$ and $k \geq 2$.

Problem 3.2. Find the thickness of $K_{n, n+4 k+2}$ for odd $n$ and $k \geq 0$.

\section{Acknowledgement}

We are grateful to the two anonymous referees for their helpful comments. The second author is supported by the NNSFC under Grant No. 11471106.

\section{References}

[1] A. Aggarwal, M. Klawe and P. Shor, Multilayer grid embeddings for VLSI, Algorithmica, 6 (1991), 129-151.

[2] V. B. Alekseev and V. S. Gonchakov, Thickness of arbitrary complete graphs, Mat. Sb., 101 (No. 143) (1976), 212-230 
[3] G. D. Battista, P. Eades, R. Tamassia and I. G. Tollis, Graph drawing, Prentice-Hall, Englewood Cliffs, NJ, 1999.

[4] J. Battle, F. Harary and Y. Kodama, Every planar graph with nine points has a nonplanar complement, Bull. Amer. Math. Soc. 68 (1962), 569-571.

[5] L. W. Beineke and F. Harary, The thickness of the complete graph, Canad. J. Math., 17 (1965), 850-859.

[6] L. W. Beineke, F. Harary and J.W. Moon, On the thickness of the complete bipartite graph, Proc. Cambridge Philos. Soc., 60 (1964), 1-5.

[7] L. W. Beineke and R. J. Wilson, Topics in topological graph theory, With the collaboration of Jonathan L. Gross and Thomas W. Tucker, Cambridge University Press, 2009.

[8] J. L. Gross and F. Harary, Some problems in topological graph theory, J. Graph Theory, 4 (1980), 253-263.

[9] S. Hu and Y. Chen, The thickness of some complete tripartite graphs, preprint.

[10] S. Isao and H. Ozaki, On the planar decomposition of a complete bipartite graph. Siam J. Appl. Math. 16 (1968), 408-416.

[11] A. Mansfield, Determining the thickness of graphs is NP-hard, Math. Proc. Cambridge Philos. Soc., 93 (1983), 9-23.

[12] P. Mutzel, T. Odenthal and M. Scharbrodt, The thickness of graphs: a survey, Graphs and Combin., 14 (1998), 59-73.

[13] J. M. Vasak, The thickness of the complete graph, (Doctoral dissertation, University of Illinois at Urbana-Champaign), 1976.

[14] W. T. Tutte, The non-biplanar character of the complete 9-graph. Canad. Math. Bull., (1963), 319-330.

[15] W. T. Tutte, The thickness of a graph, Indag. Math., 25 (1963), 567-577.

[16] Y. Yang, Remarks on the thickness of $K_{n, n, n}$, Ars Math. Contemp. 12 (2017), 135-144. 
A A planar decomposition $\left\{G_{1}, G_{2}, G_{3}, G_{4}, G_{5}\right\}$ for $K_{17,21}$

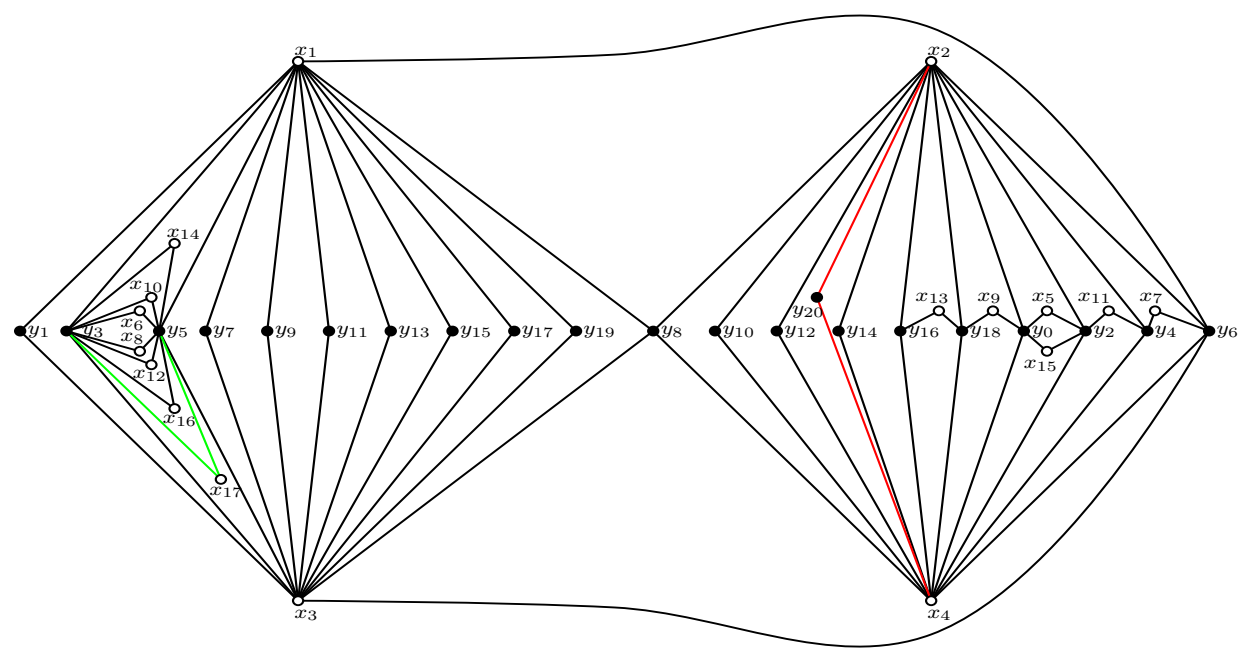

Figure 3: The Graph $G_{1}$

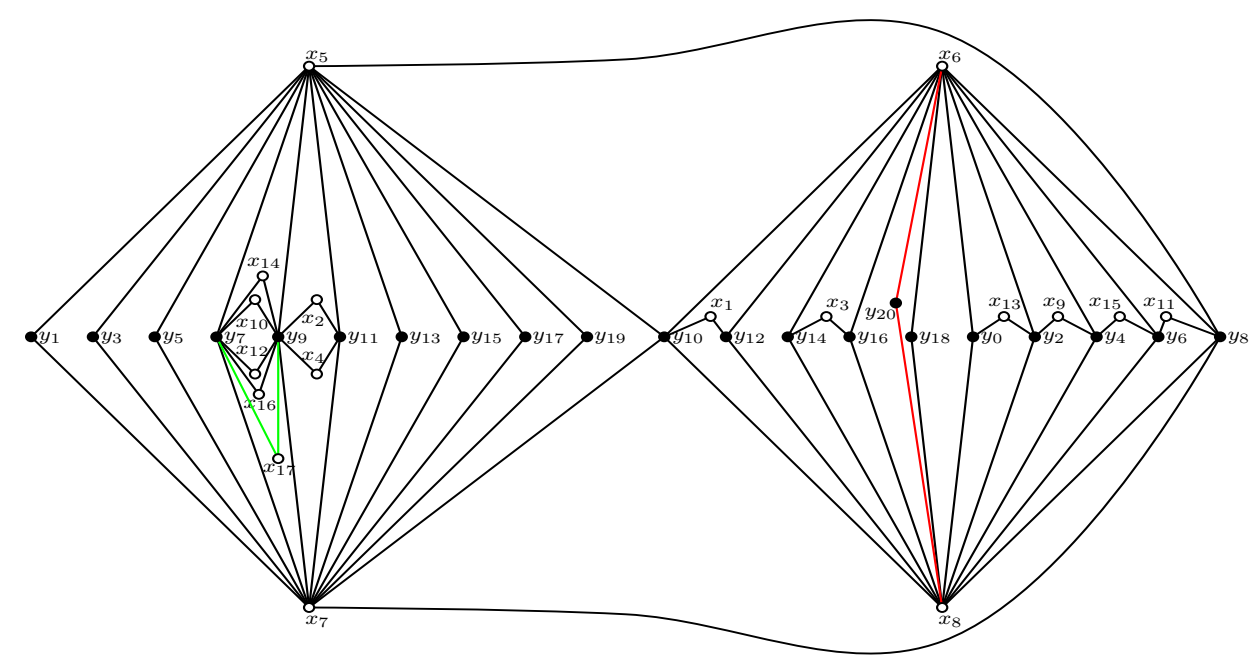

Figure 4: The Graph $G_{2}$ 


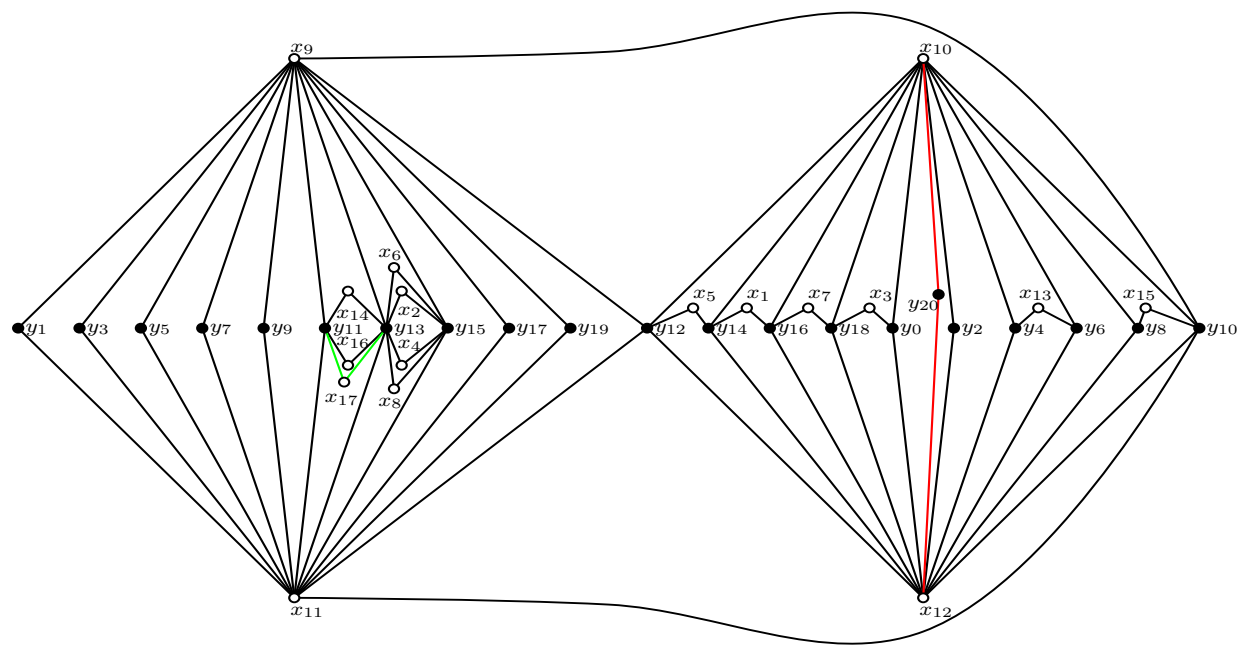

Figure 5: The Graph $G_{3}$

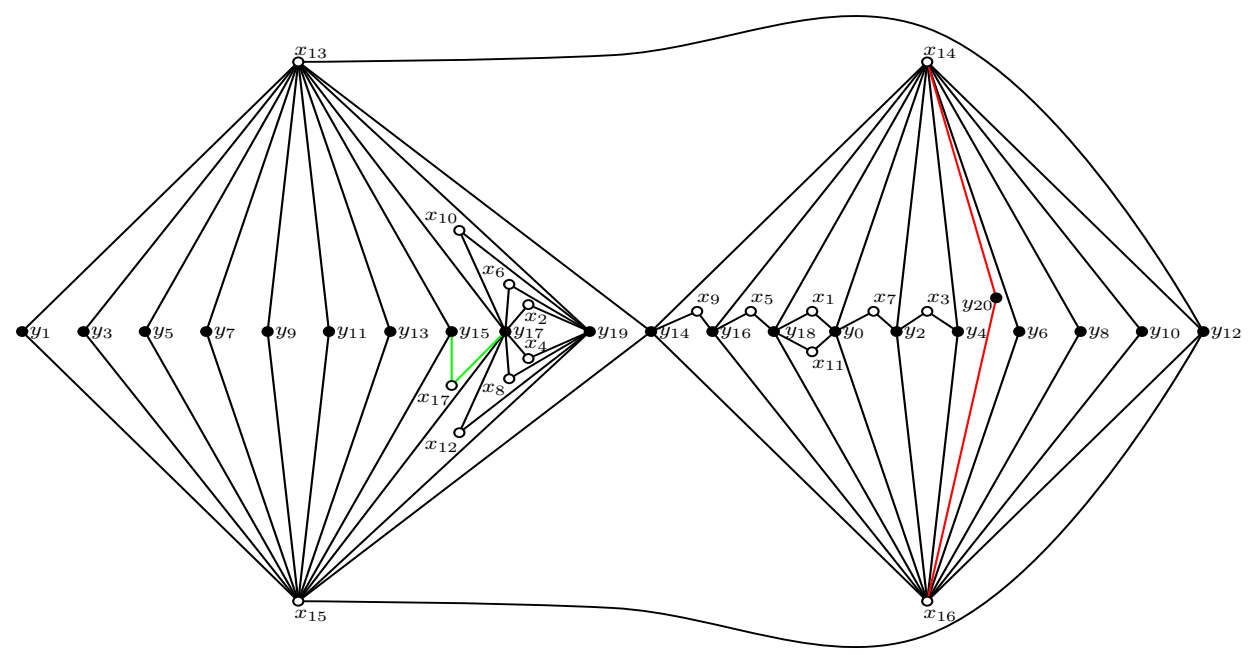

Figure 6: The Graph $G_{4}$ 


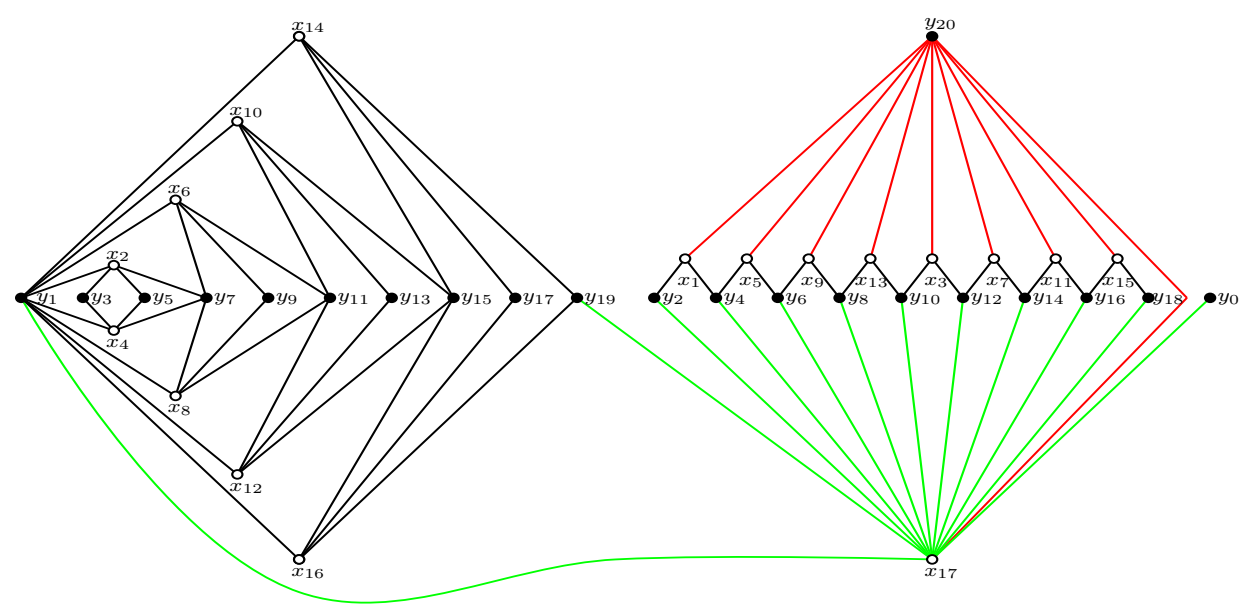

Figure 7: The Graph $G_{5}$ 
B A planar decomposition $\left\{G_{1}, G_{2}, G_{3}, G_{4}, G_{5}, G_{6}\right\}$ for $K_{21,25}$

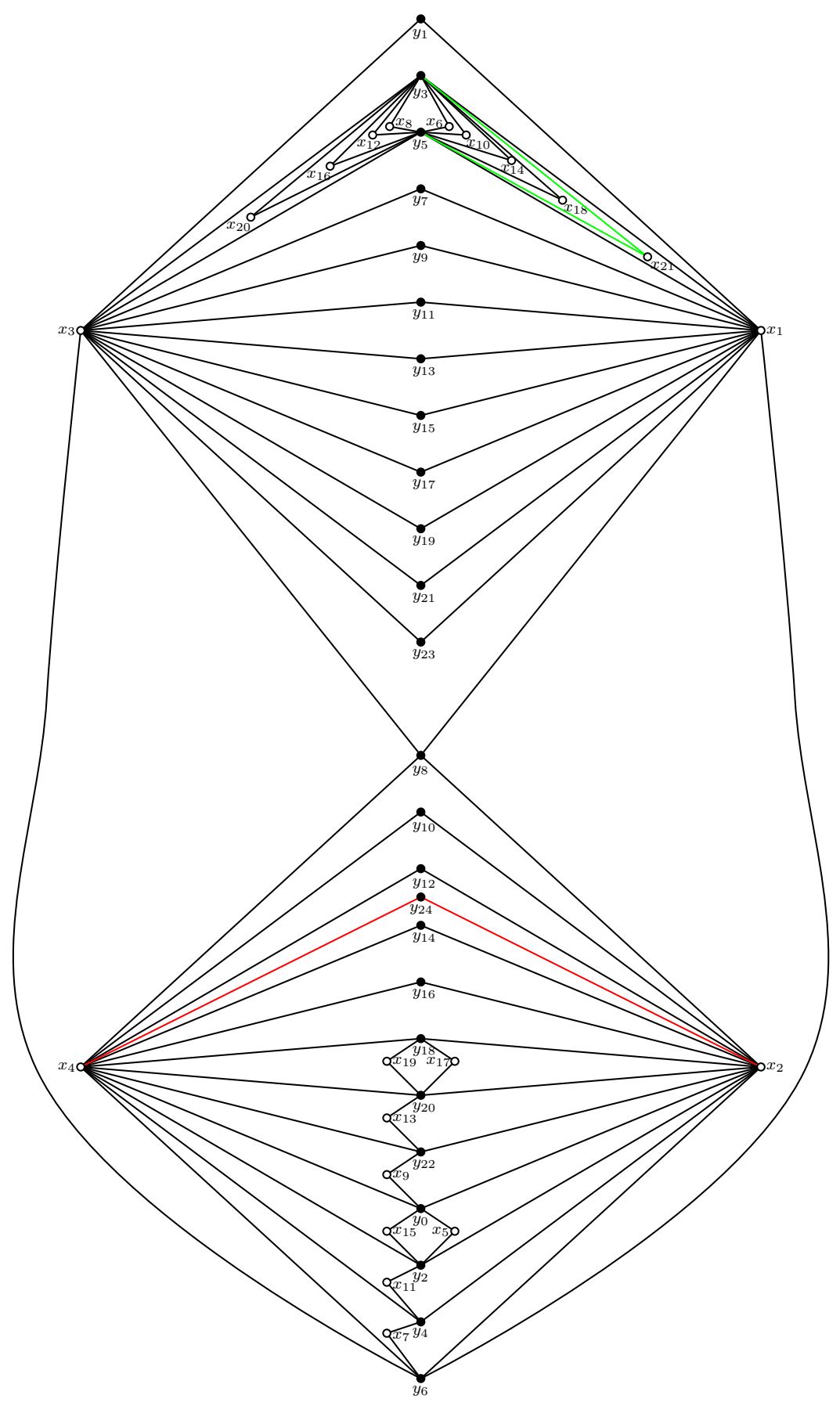

Figure 8: The Graph $G_{1}$ 


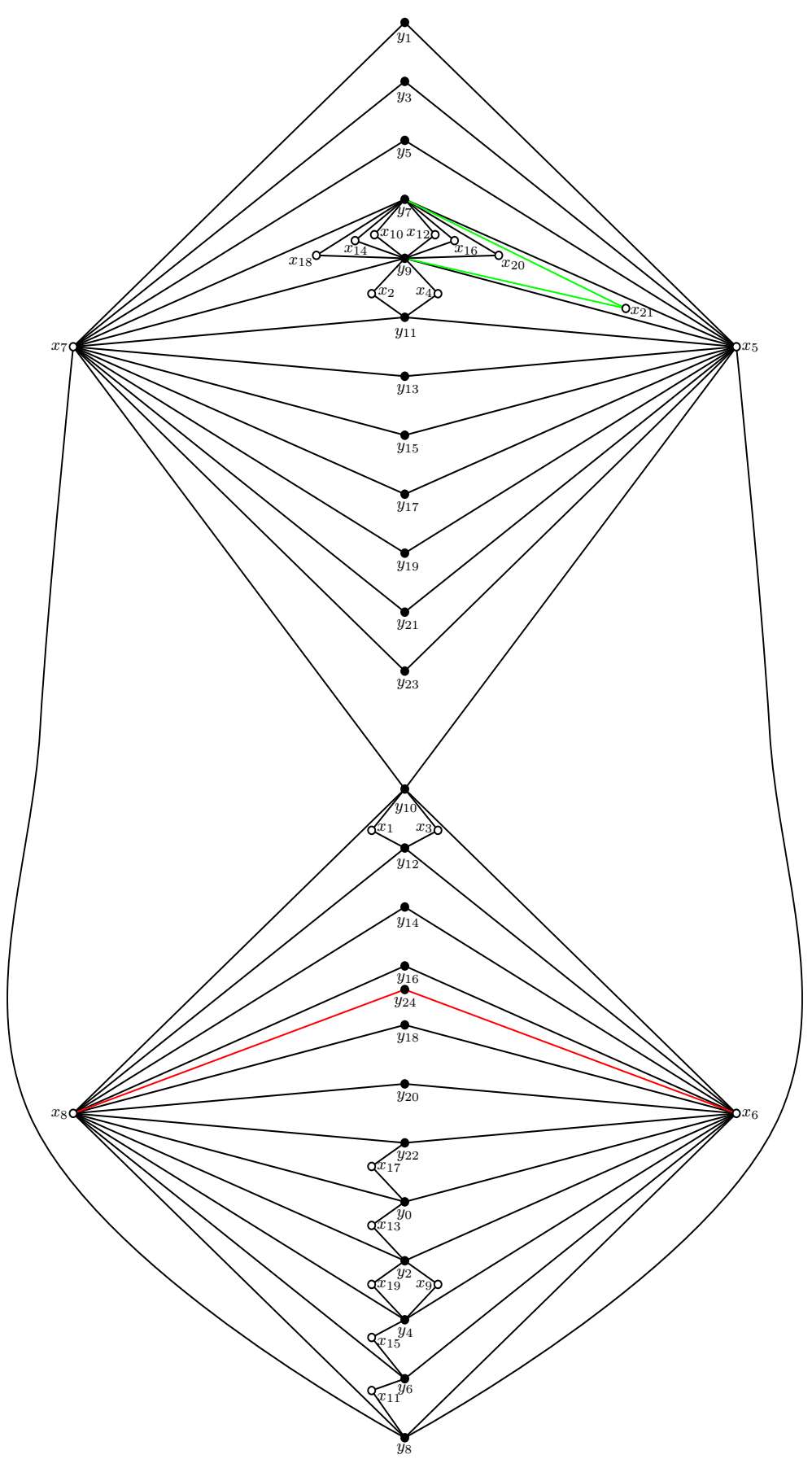

Figure 9: The Graph $G_{2}$ 


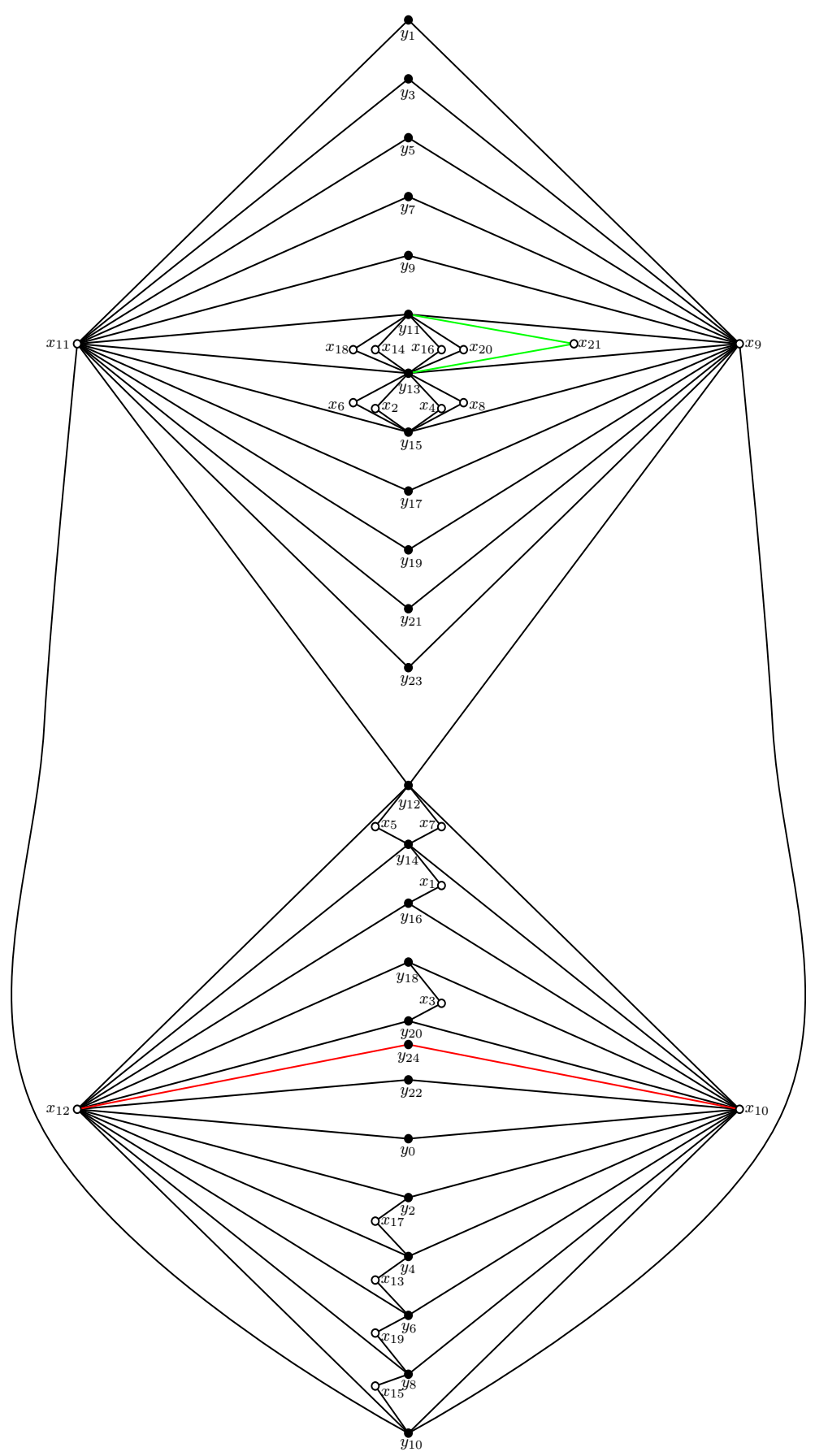

Figure 10: The Graph $G_{3}$ 


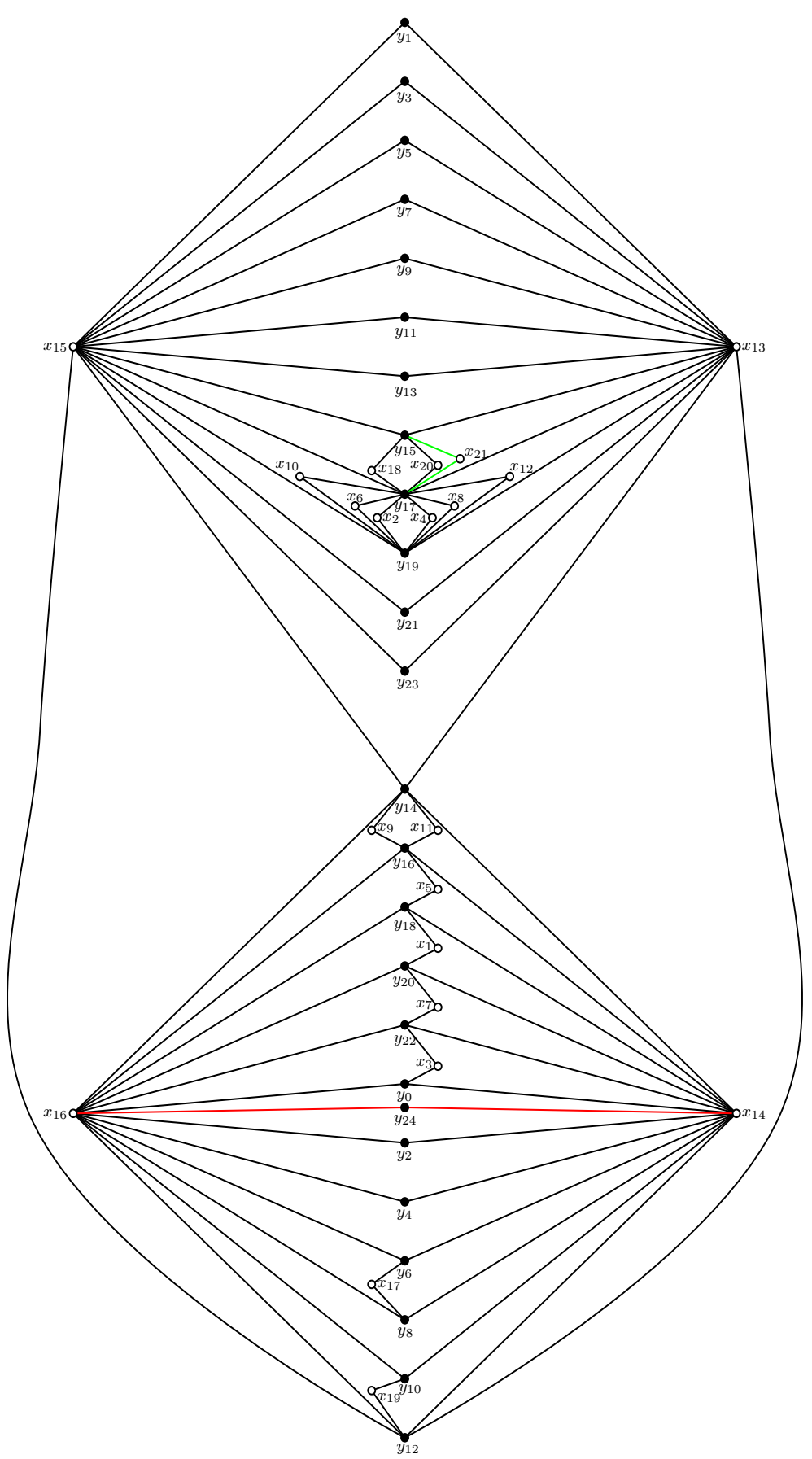

Figure 11: The Graph $G_{4}$ 


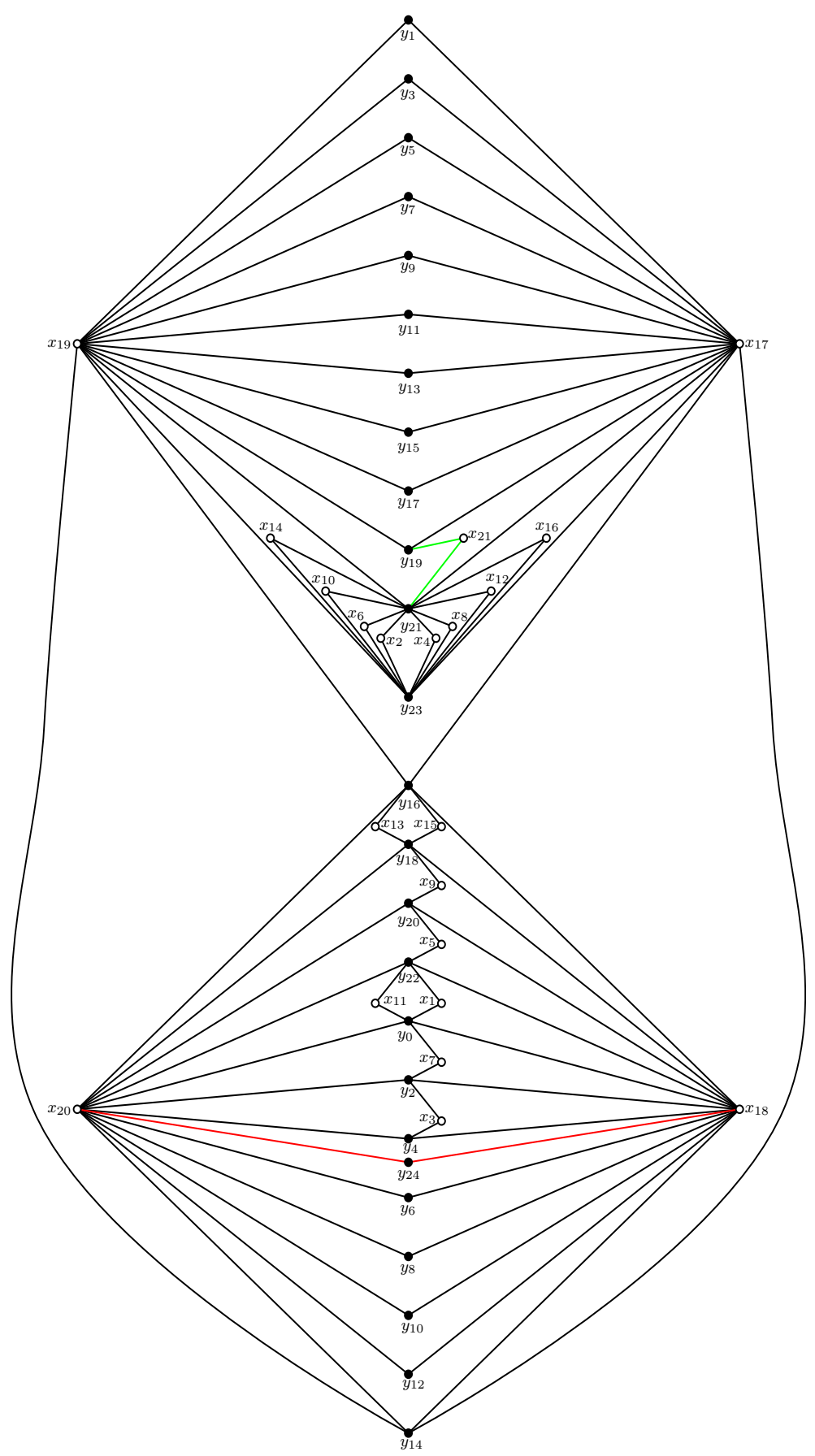

Figure 12: The Graph $G_{5}$ 


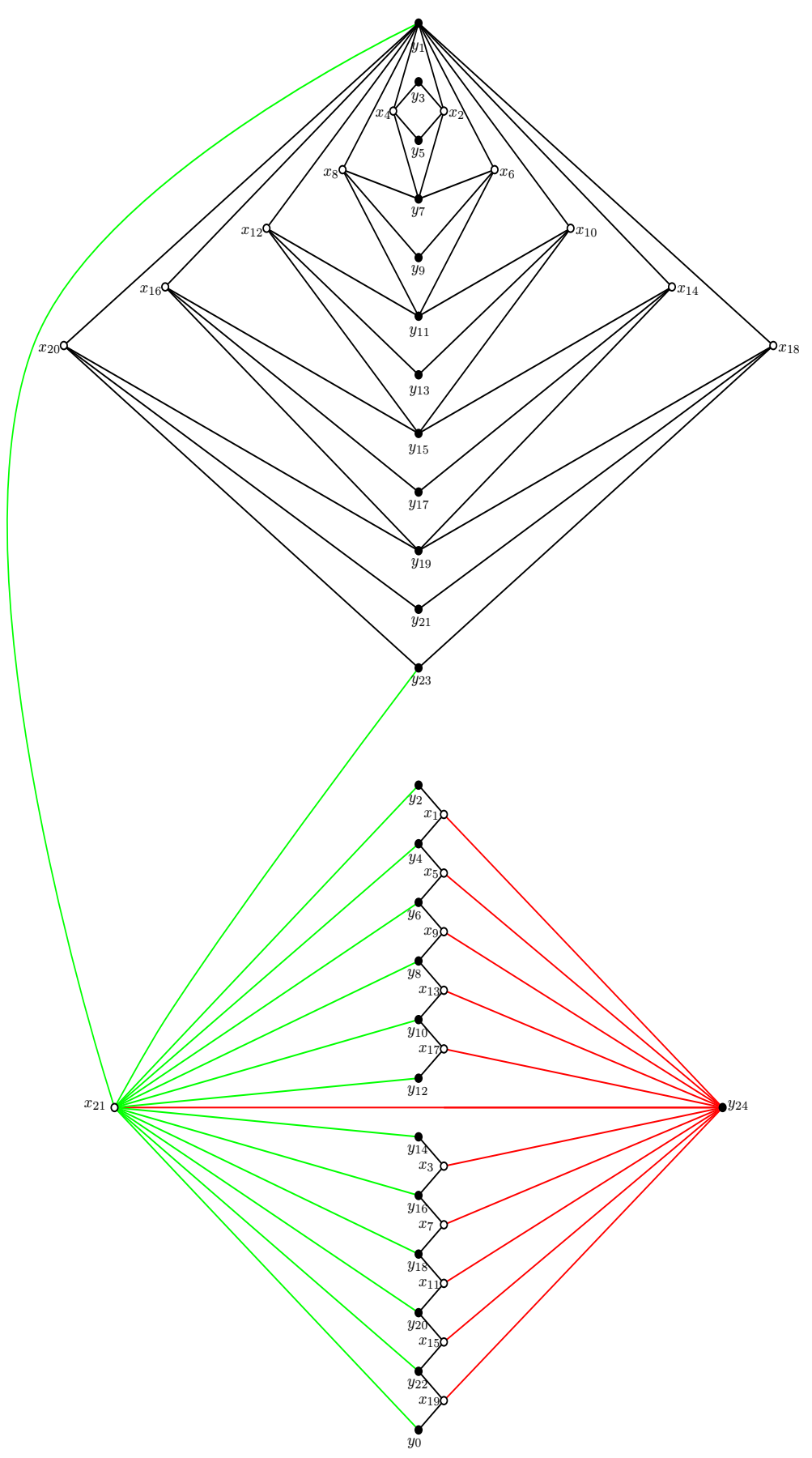

Figure 13: The Graph $G_{6}$ 